\title{
Decreased Risk of Influenza in Child and Adolescent Patients with Attention-Deficit Hyperactivity Disorder Following Methylphenidate Treatment: A Nationwide Cohort Study in Taiwan
}

This article was published in the following Dove Press journal:

Neuropsychiatric Disease and Treatment

Hsuan Lee, (iD) ${ }^{1} *$ Vincent Chin-Hung Chen, ${ }^{2,3 * *}$ Yao-Hsu Yang, (D) ${ }^{4-6}$ Ting-Yu Kuo, ${ }^{4}$ Tzu-Chin Lin, ${ }^{7,8}$ Shu-I Wu, ${ }^{9,10}$ KaiLiang Kao, ${ }^{11,12}$ Jun-Cheng Weng, (ID) ${ }^{3,13}$ Brent Allan Kelsen, (iD) ${ }^{14,15}$ Sophie Hsin-Yi Liang (iD) 2,16

'Department of Psychiatry, Taipei Veterans General Hospital, Taipei, Taiwan; ${ }^{2}$ Department of Medicine, Chang Gung University, Taoyuan, Taiwan; ${ }^{3}$ Department of Psychiatry, Chang Gung Memorial Hospital at Chiayi,Chiayi, Taiwan; ${ }^{4}$ Health Information and Epidemiology Laboratory of Chang Gung Memorial Hospital, Chiayi Branch, Chiayi,Taiwan; ${ }^{5}$ Department of Traditional Chinese Medicine, Chang Gung Memorial Hospital,Taiwan; ${ }^{6} \mathrm{~S}$ chool of Traditional Chinese Medicine, College of Medicine, Chang Gung University, Taoyuan, Taiwan; ${ }^{7}$ Bethel Psychiatric Clinic, Taipei, Taiwan; ${ }^{8}$ Institute of Science, Technology and Society, National Yang-Ming University Taipei, Taiwan; ${ }^{9}$ Mackay Memorial Hospital, Section of Psychiatry, Taipei, Taiwan; ${ }^{10}$ Department of Medicine, Mackay Medical College, Taipei, Taiwan; "Department of Pediatrics, Far Eastern Memorial Hospital, Taipei, Taiwan;

${ }^{12}$ Department of Industrial Management, Oriental Institute of Technology, Taipei, Taiwan;

${ }^{13}$ Department of Medical Imaging and Radiological Sciences, Chang Gung University, Taoyuan, Taiwan; ${ }^{14}$ Language Center, National Taipei University, New Taipei City, Taiwan;

${ }^{15}$ Department of Psychology, Auckland University of Technology, Auckland, New Zealand;

${ }^{16}$ Department of Child Psychiatry, Chang Gung

Memorial Hospital at Taoyuan, Taoyuan, Taiwan

*These authors contributed equally to this work

Correspondence: Sophie Hsin-Yi Liang Department of Child Psychiatry, Chang Gung Memorial Hospital at Taoyuan, No.

123. Dinghu Road, Guishan Township,

Taoyuan 333, Taiwan

Email sophie.Ihy@gmail.com
Background: Young individuals with attention-deficit hyperactivity disorder (ADHD) may have an elevated risk of influenza because of the difficulty in complying with the behavioral procedures that help protect against influenza. Moreover, the effects of sufficient methylphenidate treatment on influenza have received little attention.

Objective: This study evaluated the association between ADHD medication usage and influenza and assessed the effect of duration of ADHD treatment on the risk of influenza using a nationwide population-based database.

Methods: This study investigated methylphenidate usage and the risk of influenza among children and adolescents with ADHD. We identified 5259 young individuals aged less than 18 years who were diagnosed as having ADHD between 1996 and 2013 from the National Health Insurance Research Database of Taiwan, and we tested whether methylphenidate use affects influenza risk using Cox proportional hazard models.

Results: After controlling for confounding factors, the results indicated that influenza risk significantly reduced in the group of ADHD patients who were prescribed methylphenidate for 90 days and more (hazard ratio [HR]: 0.62, 95\% confidence interval [CI]: 0.52-0.75, $p<0.001$ ), demonstrating a $38 \%$ reduction in the risk of influenza in this group. However, this was not observed in the group of ADHD patients who used methylphenidate for 1-90 days (HR: $0.69,95 \%$ CI: $0.89-1.05, p=0.12$ ).

Conclusion: The lower incidence of influenza observed in the group prescribed with methylphenidate for a longer period highlights the importance of compliance to medication and psychoeducation with regard to ADHD management.

Keywords: ADHD, influenza, methylphenidate

\section{Introduction}

Attention-deficit hyperactivity disorder (ADHD) is the most common childhood neurodevelopmental disorder and typically affects approximately 5-7\% of the pediatric population irrespective of the geographical location or cultural background. ${ }^{1-3}$ People with ADHD typically demonstrate enduring hyperactivity, impulsivity, reduced attention, and increased distractibility that may span from childhood to adulthood and potentially impair everyday functioning. ${ }^{4}$ This disorder is associated with delayed social and emotional development, ${ }^{5}$ increased likelihood of self-harm and suicide attempts, ${ }^{6,7}$ increased risk of accident and injury, ${ }^{8}$ and high 
family stress and healthcare utilization. ${ }^{9}$ Studies have indicated that pharmacological treatment, including stimulant medication or atomoxetine, can improve functional outcomes and health-related quality of life, ${ }^{10,11}$ and pharmacological treatment is associated with a lower rate of substance-related problems ${ }^{12}$ and risk-related behaviors including suicide, ${ }^{13,14}$ injury ${ }^{15,16}$ and fracture. ${ }^{17}$ Another study of stimulant medications for the treatment of ADHD suggested that the medications can successfully decrease the risks of certain common psychiatric conditions, such as depression, anxiety, and personality disorder. ${ }^{18}$

Influenza is generally regarded as a severe illness only for elderly persons and those with chronic conditions that increase their risk of complications. ${ }^{19}$ However, during the novel $2009 \mathrm{H} 1 \mathrm{~N} 1$ influenza pandemic, the highest incidence rates were recorded among children and young adults, with incidence rates exceeding $40 \%$ in preschool-aged children and $30 \%$ in school-aged children. ${ }^{20}$ The burden of influenza on pediatric respiratory hospitalization is demonstrated by the rate of respiratory hospitalization attributed to influenza, which is $10 \%$ in children aged less than 18 years and $16 \%$ among children aged 5-17 years worldwide. $^{21}$ Nonpharmaceutical behavior prevention of influenza and reduction of influenza virus transmission among children and adolescents involve practicing appropriate hand hygiene (frequent hand washing with hand wash products) and upholding respiratory hygiene, ${ }^{22,23}$ cough etiquette and social distancing. ${ }^{23-25}$ In addition, psychoeducation and awareness on the risk for influenza are emphasized. ${ }^{23,24}$

Research has revealed that ADHD is associated with inflammatory and immune-related disorders (ie, atopic disorders including allergic rhinitis, asthma and eczema), ${ }^{26-28}$ suggesting a connection between psychopathology and inflammatory processes. One meta-analysis revealed that ADHD patients had increased rates of asthma. ${ }^{29}$ Moreover, the odds of allergic rhinitis, eczema and allergic conjunctivitis in children with ADHD were found to be slightly higher than the non-ADHD group. ${ }^{29}$ Although sufficient evidence has not been obtained to demonstrate that influenza is related to atopic diseases ${ }^{30,31}$ they have been recognized as predisposing factors for upper respiratory infections and symptom deterioration. In addition, the neurocognitive deficits associated with ADHD, for instance, difficulty following instructions and protocols or impulsive touching, which might hinder compliance with hand hygiene, could possibly lead to infectious diseases. ${ }^{32}$ In addition, a high percentage of children and adolescents with ADHD has nail-biting problems, which may also increase the risk of infections. ${ }^{33,34}$
To the best of our knowledge, no study has investigated the relationship between the effect of ADHD medication usage toward the risk of influenza. Therefore, to bridge this gap in the literature, we evaluated the association between ADHD medication usage and influenza and the effect of duration of ADHD treatment on the risk of influenza using a nationwide population-based database.

\section{Methods}

\section{Data Sources}

Taiwan's NHI program was established by the government in March 1995, and this single-payer national health insurance program for healthcare delivery covered over $92 \%$ of the national population at that time. The government supported NHI program centralized healthcare utilizations, including inpatient, outpatient, and dental care. In 2009, the coverage of the NHI program increased to $99.5 \%$ of all medical claims. From the National Health Insurance Research Database of Taiwan (NHIRD-TW), the Bureau of NHI established a representative database of $1,000,000$ people (ie, approximately $5 \%$ of the population of Taiwan) randomly sampled from the year 2005 registry of all NHI enrollees for research purposes, forming the Longitudinal Health Insurance Database (LHID). No statistically significant differences are observed in gender, age, or healthcare cost between the aforementioned LHID sample and all NHI enrollees. ${ }^{35}$

\section{Ethics}

The LHID contains no identifying information. This study was approved by the Institutional Review Board of Chang Gung Memorial Hospital.

\section{Study Population and Outcome Event}

In this study, we identified 9826 patients newly diagnosed as having ADHD who either met criteria of DSM-IV ${ }^{36}$ or DSMIV-TR ${ }^{37}$ within the follow-up period between January 31, 1996 and December 31, 2013. In this ADHD cohort, the diagnosis of ADHD was defined as follows: at least two outpatient records of ADHD within 1 year or at least one inpatient record of ADHD based on the International Classification of Diseases, Ninth Revision (ICD-9) code $314 .{ }^{38}$ No significant difference was found for the incidence rate of influenza between sex-, age-, urbanization- and income-matched ADHD and non-ADHD cohorts.

Influenza was the outcome of this study and was operationalized according to ICD-9 codes 487 and $488 .^{38}$ Enrollees diagnosed as having influenza before the 
diagnosis of ADHD $(n=1786)$ were omitted. pursuant to the Bureau of NHI guidelines, methylphenidate Furthermore, the cohort identified from the database excluded those patients born before 1996 and after 2005 $(\mathrm{n}=2756)$, and those with missing data on their residential area $(\mathrm{n}=3)$, diagnosed as having ADHD in $2013(\mathrm{n}=$ 271), or receiving influenza vaccinations (code V04.8) 1 year before enrollment $(n=68)$. The flowchart of the criteria of inclusion and exclusion of this study is presented in Figure 1.

\section{Methylphenidate Usage}

The medications approved for ADHD treatment in Taiwan are methylphenidate and atomoxetine. Although atomoxetine, a nonstimulant, was approved in Taiwan in 2007,

is the first choice of treatment for ADHD. Atomoxetine is only recommended for patients with ADHD who have unsatisfactory outcomes with methylphenidate, including poor response or intolerant side effects, or those who have concurrent conditions, including anxiety and tic disorder. In the LHID cohort, $4.3 \%$ of patients with ADHD were prescribed atomoxetine; ${ }^{39}$ therefore, we limited our analysis to methylphenidate. The study cohort was divided into three groups according to the methylphenidate use duration (0 days, 1-90 days, $>90$ days); the duration was defined as the cumulative days of methylphenidate prescription during the follow-up period. The endpoint was defined as the occurrence of influenza or the end of the study period.

9,826 patients with diagnosis of ADHD from 1996

to 2013 in NHIRD (definition of diagnosis required

at least two times outpatient follow-up or one time admission within one year)

1,786 patients had influenza prior the ADHD diagnosis were excluded.

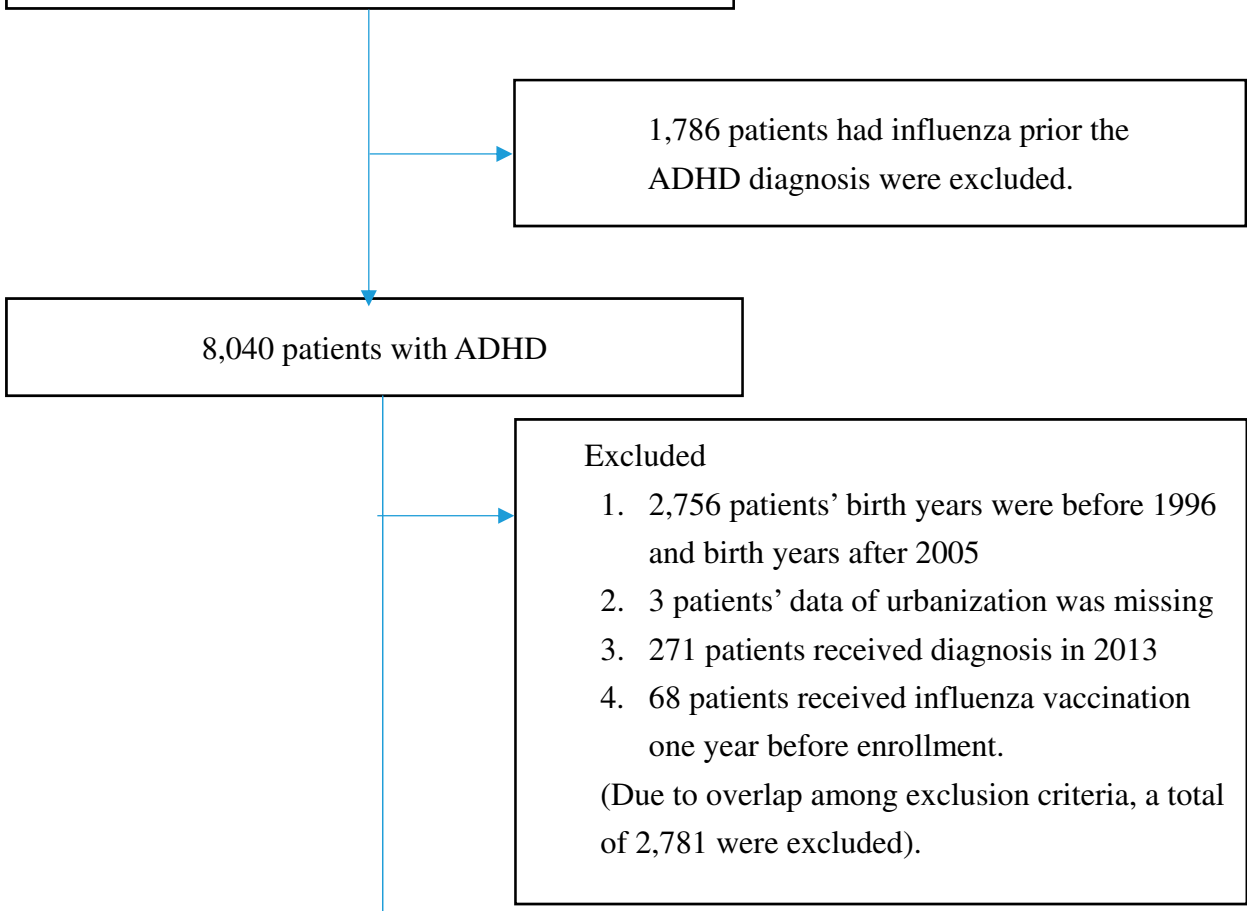

5,259 patients with ADHD aged younger than 18

years old

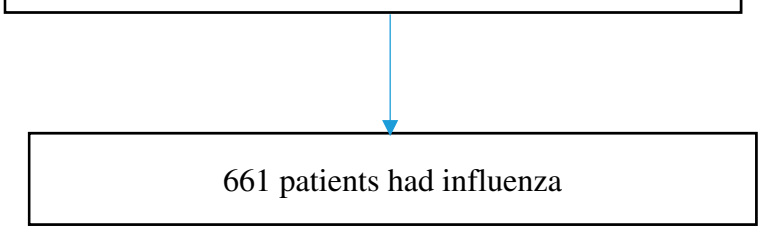

Figure I Flow diagram of criteria for inclusion and exclusion. 


\section{Statistical Analyses}

First, using the chi-square test, we examined the differences in categorical variables among the groups with no methylphenidate use (0 days), methylphenidate prescription between 1 and 90 days (1-90 days), and methylphenidate use for more than 90 days ( $>90$ days). Covariates identified from the ADHD and influenza literature were included in this study: gender, age, level of urbanization (from the most urbanized to the least urbanized), physical illness (asthma [ICD-9 code 493], atopic dermatitis [ICD9 code 691], allergic rhinitis [ICD-9 code 477], and seizure [ICD-9 code 345]), psychiatric comorbidities (pervasive developmental disorder [ICD-9 code 299] and mental retardation [ICD-9 codes 317-319]), and use of medications (sedative/hypnotic/anxiolytics). Second, to investigate the risk of influenza within this ADHD cohort, a multivariate Cox proportional hazard regression model was used to estimate the hazard ratios (HRs) with 95\% confidence intervals (CIs) while controlling for confounding factors and to compare the risk of influenza between the groups. Sensitivity analysis was performed in order to estimate the effect of longer duration (more than 180 days) usage of methylphenidate. A p value of $\leq 0.05$ indicated statistically significant differences. The SAS software program Version 9.3 (SAS Institute Inc., Cary, NC, USA) was used for data management and statistical analyses.

\section{Results}

\section{Cohort Characteristics}

We identified 5259 children and adolescents, of which $79 \%$ were male, with an incident ADHD diagnosis from the LHID between 1996 and 2013. Among this ADHD cohort, 2252 (42.8\%) individuals with ADHD had not used any ADHD medication, while 3007 participants (57.2\%) had used ADHD medication (1055 (20.1\%) had used ADHD medication between 1 to 90 days and 1952 (37.1\%) had used it for more than 90 days).

Of these, $661(12.57 \%)$ children and adolescents had diagnoses of influenza during the follow-up period. Among them, 340 (51.43\%) did not use methylphenidate, 124 (18.76\%) used methylphenidate for less than 90 days, and $197(29.80 \%)$ used methylphenidate for more than 90 days $(\mathrm{p}<0.001)$. The detailed demographic data, comorbidities, and medication use are provided in Table 1. In the groups with methylphenidate use, more patients tended to be male, older, and less urbanized compared with the group without methylphenidate use. No significant differences were observed in physical or psychiatric illness and sedative/benzodiazepine/anxiolytic medication use among the three groups (Table 1).

\section{Adjusted HRs of Influenza for Children and Adolescents with ADHD}

Table 2 presents the results of the fully adjusted Cox regression model after controlling for gender, age, level of urbanization, psychiatric or physical illnesses, and medication use (sedative/hypnotics/anxiolytics). The results indicate a statistically significant reduction in the incidence of influenza in patients with ADHD who were prescribed methylphenidate for more than 90 days (HR: $0.62,95 \%$ CI: $0.52-0.75, p<0.001)$. However, this was not observed in the group of ADHD patients who used methylphenidate for 1-90 days (HR: $0.69,95 \%$ CI: 0.89 $1.05, p=0.12$ ). An additional factor with a statistically significant association with the incidence of influenza was the comorbidity of allergic rhinitis (HR: 1.34, 95\% CI: 1.04 1.73, $p=0.021)$. The cumulative incidence of influenza according to methylphenidate prescription is presented in Figures 2 and 3.

\section{Sensitivity Analysis}

We also conducted a sensitivity analysis exploring the possible protective effects of a longer duration of methylphenidate prescription. The result of the sensitivity analysis revealed statically significant protective effects against influenza in the group with methylphenidate use for more than 180 days (HR: $0.61,95 \%$ CI: $0.51-0.74, p<0.001$ ) as shown in Supplement 1.

\section{Discussion}

To the best of our knowledge, the present study is the first to investigate the effect of psychostimulants and the moderating role of methylphenidate treatment duration on the risk of influenza among children and adolescents with ADHD. Our findings revealed that receiving methylphenidate treatment for more than 90 days was associated with an approximately $38 \%$ potentially reduction in the risk of influenza compared with no methylphenidate treatment. Furthermore, methylphenidate treatment duration shorter than 90 days did not reduce the risk of influenza. 
Table I Characteristics of ADHD Youths with and without MPH Usage

\begin{tabular}{|c|c|c|c|c|c|c|c|c|}
\hline \multirow[t]{2}{*}{ Variables } & \multicolumn{2}{|c|}{$\begin{array}{l}M P H=0 \\
\text { d }(\mathbf{N}=2252)\end{array}$} & \multicolumn{2}{|c|}{$\begin{array}{l}\text { MPH between I and 90d } \\
(N=1055)\end{array}$} & \multicolumn{2}{|c|}{$\begin{array}{l}\text { MPH >90d } \\
(\mathrm{N}=1952)\end{array}$} & \multirow[t]{2}{*}{ Chi-Square } & \multirow[t]{2}{*}{$P$ value } \\
\hline & Count & $\%$ & Count & $\%$ & Count & $\%$ & & \\
\hline Gender & & & & & & & 39.2130 & $<0.001$ \\
\hline Male & 1692 & 75.13 & 844 & 80.00 & 1619 & 82.94 & & \\
\hline Female & 560 & 24.87 & 211 & 20.00 & 333 & 17.06 & & \\
\hline Age (years) & \multicolumn{2}{|c|}{$6.76 \pm 2.68$} & \multicolumn{2}{|c|}{$8.03 \pm 2.57$} & \multicolumn{2}{|c|}{$7.66 \pm 2.44$} & \multirow[t]{4}{*}{275.2047} & \multirow[t]{4}{*}{$<0.001$} \\
\hline $0-5$ & 779 & 34.59 & 155 & 14.69 & 302 & 15.47 & & \\
\hline $6-11$ & 1342 & 59.59 & 794 & 75.26 & |49| & 76.38 & & \\
\hline $12-18$ & $13 \mid$ & 5.82 & 106 & 10.05 & 159 & 8.15 & & \\
\hline Levels of urbanization ${ }^{a}$ & & & & & & & 29.5361 & $<0.001$ \\
\hline I (Most urbanized) & 830 & 36.86 & 349 & 33.08 & 737 & 37.76 & & \\
\hline 2 & 1096 & 48.67 & 510 & 48.34 & 876 & 44.88 & & \\
\hline 3 & 245 & 10.88 & 121 & 11.47 & 220 & 11.27 & & \\
\hline 4 (Least urbanized) & 81 & 3.60 & 75 & 7.11 & 119 & 6.10 & & \\
\hline \multicolumn{9}{|l|}{ Covariates } \\
\hline Seizure & & & & & & & 2.4725 & 0.29 \\
\hline No & 2180 & 96.80 & 1022 & 96.87 & 1874 & 96.00 & & \\
\hline Yes & 72 & 3.20 & 33 & 3.13 & 78 & 4.00 & & \\
\hline Asthma & & & & & & & 0.1533 & 0.926 \\
\hline No & 1550 & 68.83 & 731 & 69.29 & 1339 & 68.60 & & \\
\hline Yes & 702 & 31.17 & 324 & 30.71 & 613 & 31.40 & & \\
\hline Mental retardation & & & & & & & 4.6313 & 0.099 \\
\hline No & 2152 & 95.56 & 998 & 94.60 & 1837 & 94.11 & & \\
\hline Yes & 100 & 4.44 & 57 & 5.40 & 115 & 5.89 & & \\
\hline ASD & & & & & & & 0.2155 & 0.898 \\
\hline No & 2159 & 95.87 & 1015 & 96.21 & 1874 & 96.00 & & \\
\hline Yes & 93 & 4.13 & 40 & 3.79 & 78 & 4.00 & & \\
\hline Sedative/hypnotics/anxiolytics & & & & & & & 2.5407 & 0.281 \\
\hline No & 1608 & 71.40 & 740 & 70.14 & 1350 & 69.16 & & \\
\hline Yes & 644 & 28.60 & 315 & 29.86 & 602 & 30.84 & & \\
\hline Atopic dermatitis & & & & & & & 0.1415 & 0.932 \\
\hline No & 900 & 39.96 & 419 & 39.72 & 769 & 39.40 & & \\
\hline Yes & 1352 & 60.04 & 636 & 60.28 & 1183 & 60.60 & & \\
\hline Allergic rhinitis & & & & & & & 0.6116 & 0.737 \\
\hline No & 1177 & 52.26 & 538 & 51.00 & 1023 & 52.41 & & \\
\hline Yes & 1075 & 47.74 & 517 & 49.00 & 929 & 47.59 & & \\
\hline Influenza & & & & & & & 24.6388 & $<0.001$ \\
\hline No & 1912 & 84.90 & 931 & 88.25 & 1755 & 89.91 & & \\
\hline Yes & 340 & 15.10 & 124 & 11.75 & 197 & 10.09 & & \\
\hline Follow-up Years & \multicolumn{2}{|c|}{$5.16 \pm 3.27$} & \multicolumn{2}{|c|}{$4.75 \pm 3.07$} & \multicolumn{2}{|c|}{$5.53 \pm 2.86$} & & $<0.001$ \\
\hline
\end{tabular}

Note: ${ }^{2}$ Residential area are subgrouped by urbanized level.

Abbreviations: ASD, autism spectrum disorder; MPH, methylphenidate. 
Table 2 Cox's Proportional Hazards Model for Influenza in ADHD Youths

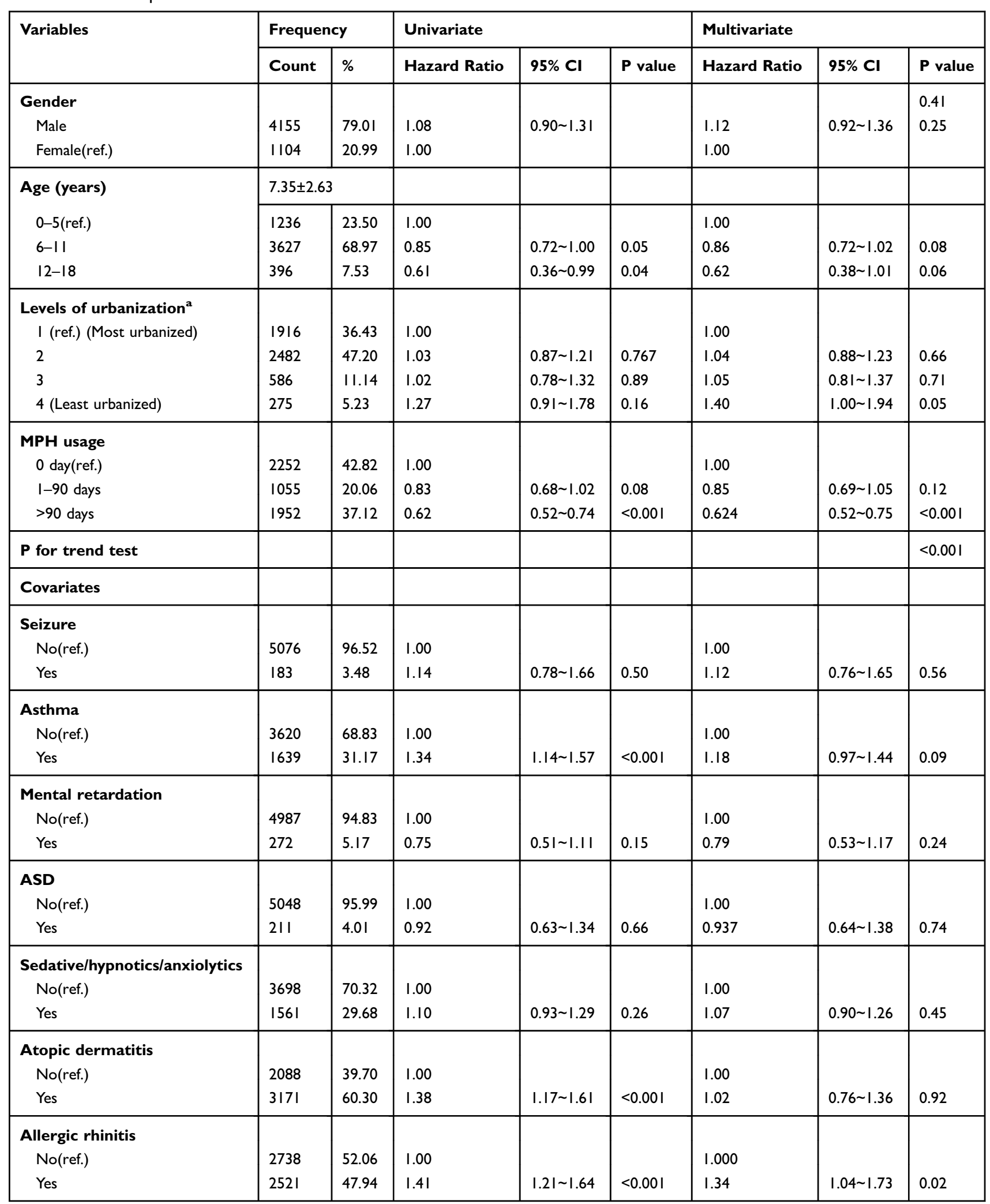

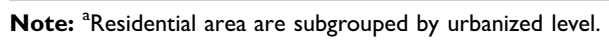

Abbreviations: ASD, autism spectrum disorder; MPH, methylphenidate. 


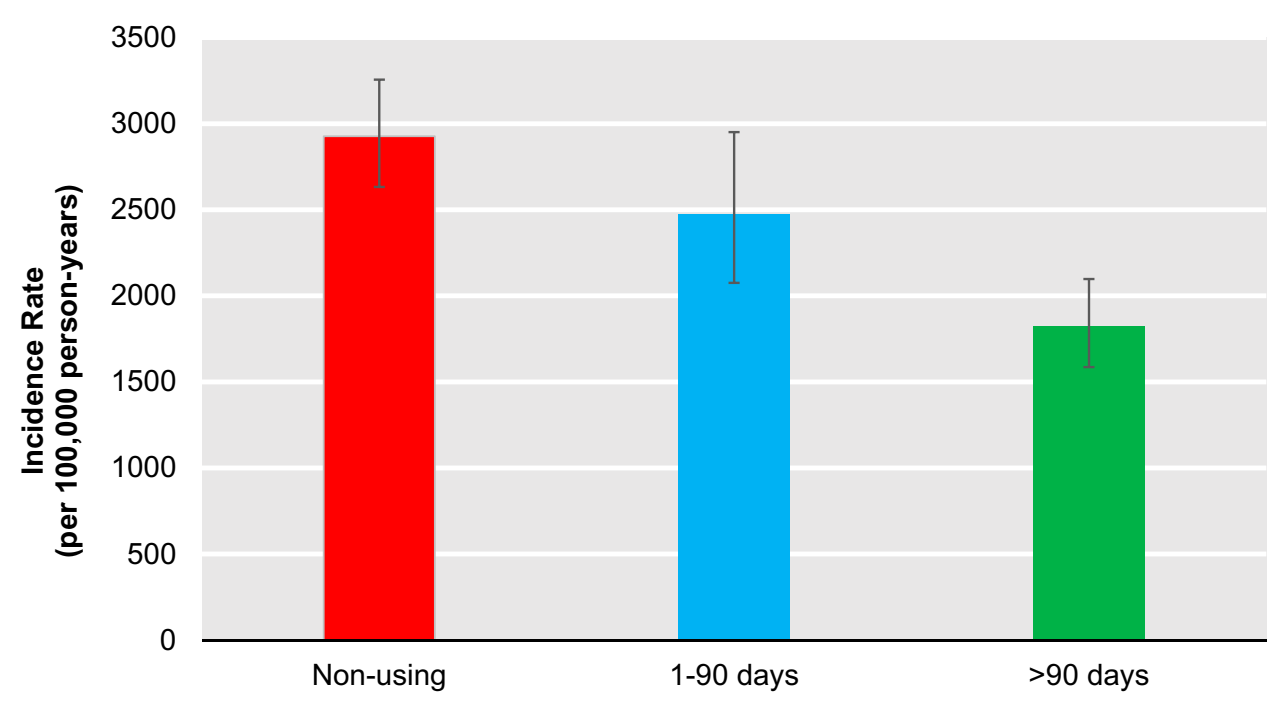

Figure 2 Incidence rates of influenza according to medication status in 5259 ADHD youths in Taiwan. ${ }^{\mathrm{a}}$ Note: ${ }^{a}$ Error bars indicate $95 \%$ confidence intervals.

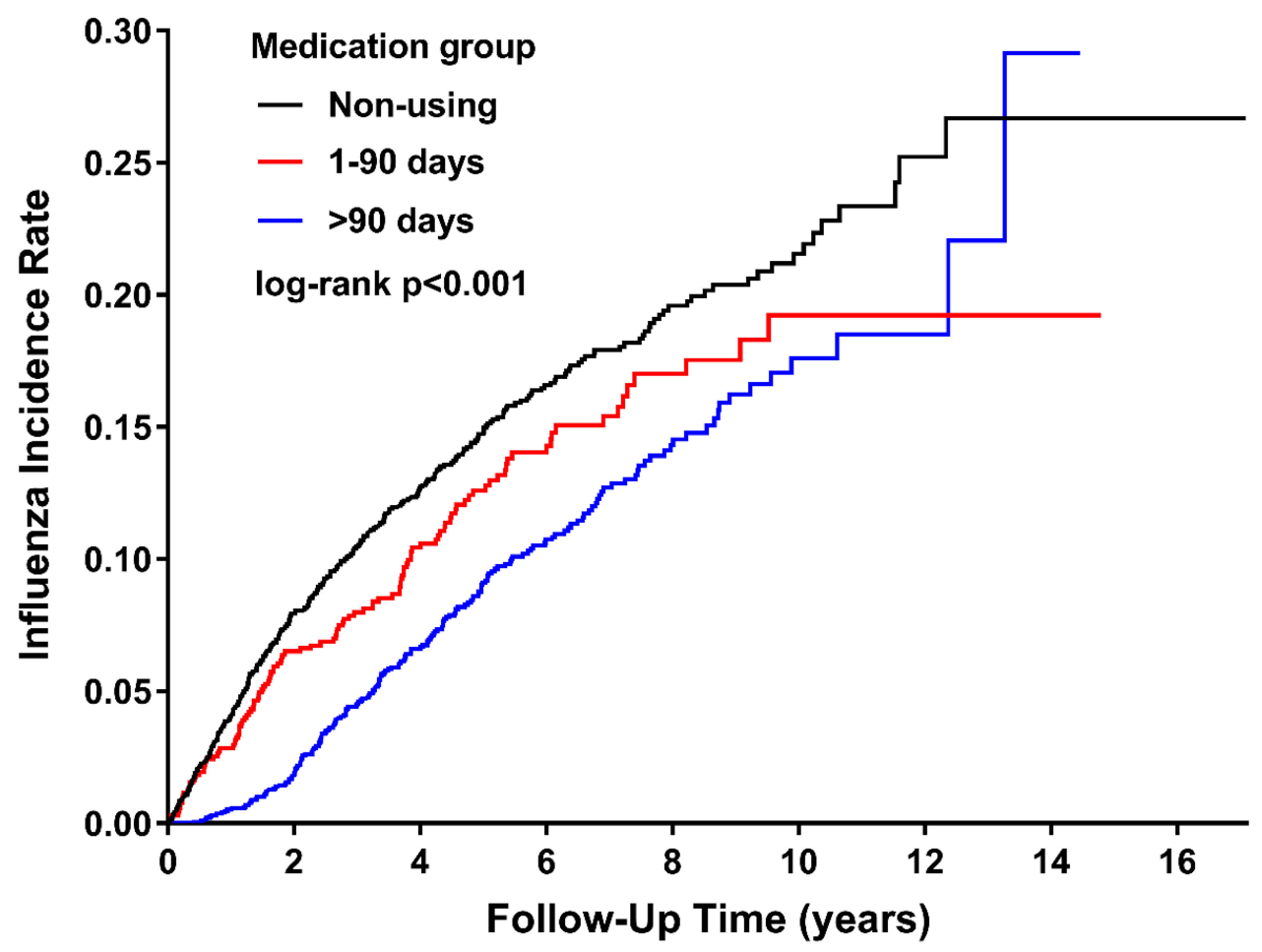

Gray's Test for Equality of Cumulative Incidence Functions

\begin{tabular}{|r|r|r|}
\hline Chi-Square & DF & Pr $>$ Chi-Square \\
\hline 29.2667 & 2 & $<.0001$ \\
\hline
\end{tabular}

Figure 3 Cumulative incidence functions of influenza according to medication status in 5259 ADHD youths in Taiwan. 


\section{Duration of Methylphenidate Use and Influenza Risk}

No study has explored either the risk of ADHD for influenza nor the association between ADHD treatment and the incidence of influenza; therefore, we cannot compare our results with any study. We conduct a sensitivity analysis to explore whether ADHD is a risk factor for influenza in children and adolescents in this LHID. Although there was no statistical difference between children and adolescents with ADHD and without ADHD, the ADHD group showed slightly increased risk of influenza compared to the non-ADHD group (incidence rate of ratios, IRR=1.04; $95 \%$ CI $0.981 .11, p=0.21$ ). Future study is suggested to exam the association between ADHD and influenza.

In our study, we found the longer ADHD treatment in youths with ADHD potentially decreased the risk of influenza. At the individual level, influenza prevention involves self-protective behaviors, including hand hygiene and respiratory hygiene/cough etiquette, and these might be more easily achieved during ADHD treatment. ${ }^{40,41}$ In addition to the possible decrease in the severity of core ADHD symptoms, proper social distancing ${ }^{25}$ in conjunction with improved interpersonal, psychological, social, and educational functioning as well as improved healthrelated quality of life ${ }^{11}$ may also partly underlie this protective effect.

\section{Catecholamines and the Immune System}

Oades et al reported that elevated interleukin (IL) levels in medicated children with ADHD tended to decrease within the normal range, indicating that minor immunological system imbalances improved following medication. ${ }^{42}$ Another possible mechanism for the reduction in influenza risk caused by methylphenidate use comprises stress-modulated immune responses through the hypothalamus-pituitary-adrenal axis and sympathetic nerve system. ${ }^{43,44}$ Several studies have suggested that the release of catecholamines (epinephrine and norepinephrine) during stress may induce immune protection, which accelerates wound healing and enhances metabolic rates and resistance to infections. ${ }^{43,44}$ Bigler et al reported that methylphenidate induces the recruitment of natural killer (NK) cells owing to increased epinephrine levels. ${ }^{45}$ Their result suggested that the epinephrine-dominant stress response might promote immunological responses to induce the maturation of NK cells in peripheral circulation.
Research has described how NK cells identify influenzainfected cells with NKp44 and NKp46 receptors and help release cytotoxic enzymes to lyse these infected cells. ${ }^{46}$ It is also plausible that the reduction in the risk of influenza associated with methylphenidate use is associated with the role of NK cells against viral infection or replication. Additional studies are warranted to explore this possible biological mechanism.

\section{Allergic Rhinitis and Influenza}

In our study, patients with allergic rhinitis also had an increased tendency of contracting influenza. Abnormal immune responses to viral infections in patients with chronic respiratory illnesses such as allergic rhinitis and asthma have been detailed. ${ }^{47,48}$ Such infection, via synergistic effects with Th2-related allergic inflammation, potentially plays a role in the initiation and exacerbation of allergic rhinitis and asthma. Direct aggravation of airway impairment as a result of heightened sensitivity and through indirect immunopathologic responses of respiratory mucosal immunity represents two mechanisms through which viruses may affect the respiratory tracts of asthmatics. ${ }^{49}$

Moreover, high associations between ADHD and atopic diseases (ie allergic rhinitis, asthma and eczema) have been reported. ${ }^{26-28}$ For instance, a study revealed higher serum concentrations of various proinflammatory cytokines as well as IL-2, IL-6, IL-10, IL-13, IL-15, and interferon gamma in patients with ADHD compared with those in controls. ${ }^{26}$ Further research on the associations between ADHD, atopic diseases, and influenza is warranted.

\section{Strengths and Limitations Strengths}

The strengths in this study encompass the nationwide sample, the longitudinal cohort study nature, the clinicbased diagnosis of ADHD, explicit drug information, and varied dimensional covariates considered, together with sociodemographic, mental health factors, general health condition, and other medication use. In addition, high validity has been reported for the clinical diagnosis of ADHD in the LHID. ${ }^{35}$ Both selection bias and recall bias were diminished by the use of this longitudinal populationbased database.

\section{Limitations}

First, this study had considerable limitations relating to the lack of other personal information associated with 
the risk of influenza, such as the individual's body weight, compliance to methylphenidate use, efficacy of psychoeducation, or behavior therapy as well as personal hygiene, such as hand washing, or respiratory hygiene for influenza prevention. Second, given that the prescription of atomoxetine for ADHD is rare in Taiwan $(4.3 \%),{ }^{39}$ we only included patients who were prescribed methylphenidate and excluded those taking atomoxetine or a combination of methylphenidate and atomoxetine. However, our sensitivity analysis including patients who used atomoxetine revealed a similar protective effect for atomoxetine. Third, Taiwan's seasonal influenza vaccination program conducted by the Taiwan Centers for Disease Control commenced in 1998 and continued to be implemented in subsequent years. ${ }^{50}$ In 2013, although the seasonal influenza vaccination coverage was up to $71.98 \%$ in primary school students, ${ }^{51}$ the status of vaccination is not fully recorded in the NHIRD-TW because data of those who received the vaccination through in-school influenza vaccination programs (for school-aged children between 7 and 15 years) and through self-paid influenza vaccinations in hospitals are not included. Therefore, we were unable to identify the immunization status of all study patients.

One possible explanation for the absence of a riskreduction effect in young individuals with ADHD who did not receive methylphenidate treatment or who received methylphenidate treatment for less than 90 days may relate to nonadherence to ADHD medication and the reduced vaccination rate among the children of certain parental groups. Gau et al revealed that higher parental education (college or above) increased the risk of poor adherence to ADHD medication in Taiwan. ${ }^{52}$ One study in Brazil indicated that the vaccination coverage decreased among children in the highly educated class in some of Brazil's urban centers, indicating a shift from vaccination accessibility to vaccination acceptability regarding vaccine endorsement, particularly among those from highly educated families. ${ }^{53,54}$ Therefore, we infer less influenza vaccination coverage among non- or less medicated young individuals with ADHD in Taiwan, which may be a risk factor for the incidence of influenza.

\section{Conclusions}

The present study was based on data from a nationwide population-based database and revealed a reduction of influenza risk in children and adolescents with ADHD who were prescribed methylphenidate for periods exceeding 90 days. This protective effect was not observed among those who used methylphenidate for less than 90 days. These results indicated that prescribing the appropriate ADHD medication and improving long-term pharmacological compliance may not only reduce the core symptoms of ADHD but also reduce the risk of influenza infection. Finally, the findings point to the importance of complying with medication, including the usage duration, for individuals prescribed with stimulants for ADHD treatment.

\section{Abbreviations}

ADHD, attention-deficit hyperactivity disorder; ASD, autism spectrum disorder; NHIRD-TW, National Health Insurance Research Database of Taiwan; LHID, Longitudinal Health Insurance Database; HR, hazard ratio; CI, confidence interval; MPH, methylphenidate; IL, interleukin.

\section{Acknowledgments}

The authors are grateful for the comments and data analysis assistance provided by the Health Information and Epidemiology Laboratory (CLRPG6G0042, CLRPG6G0043). The present study was supported by a grant from Chang Gung Memorial Hospital, Chia-Yi Branch, and based on the NHIRD-TW provided by the Bureau of NHI, the Department of Health, and managed by the National Health Research Institutes. The interpretation and conclusions contained herein do not represent those of the Bureau of NHI, Department of Health, or National Health Research Institutes. Hsuan Lee and Vincent Chin-Hung Chen are co-first authors for this study.

\section{Disclosure}

VCHC has been an investigator in a clinical trial from Orient Pharma. SHYL has received speaking honoraria from Eli Lilly and Janssen. The authors report no other conflicts of interest in this work.

\section{References}

1. Polanczyk GV, Willcutt EG, Salum GA, Kieling C, Rohde LA. ADHD prevalence estimates across three decades: an updated systematic review and meta-regression analysis. Int $J$ Epidemiol. 2014;43 (2):434-442. doi:10.1093/ije/dyt261

2. Thomas R, Sanders S, Doust J, Beller E, Glasziou P. Prevalence of attention-deficit/hyperactivity disorder: a systematic review and meta-analysis. Pediatrics. 2015;135(4):e994-e1001. doi:10.1542/ peds.2014-3482 
3. Faraone SV, Larsson H. Genetics of attention deficit hyperactivity disorder. Mol Psychiatry. 2019;24:562-575. doi:10.1038/s41380-0180070-0

4. Wilens TE, Biederman J, Spencer TJ. Attention deficit/hyperactivity disorder across the lifespan. Annu Rev Med. 2002;53(1):113-131. doi:10.1146/annurev.med.53.082901.103945

5. Buitelaar J, Medori R. Treating attention-deficit/hyperactivity disorder beyond symptom control alone in children and adolescents: a review of the potential benefits of long-acting stimulants. Eur Child Adoles Psy. 2010;19(4):325-340. doi:10.1007/s00787-0090056-1

6. Impey M, Heun R. Completed suicide, ideation and attempt in attention deficit hyperactivity disorder. Acta Psychiatr Scand. 2012;125 (2):93-102. doi:10.1111/j.1600-0447.2011.01798.x

7. Conejero I, Jaussent I, Lopez R, et al. Association of symptoms of attention deficit-hyperactivity disorder and impulsive-aggression with severity of suicidal behavior in adult attempters. Sci Rep. 2019;9 (1):4593. doi:10.1038/s41598-019-41046-y

8. Chang Z, Lichtenstein P, D’Onofrio BM, Sjolander A, Larsson H. Serious transport accidents in adults with attention-deficit/hyperactivity disorder and the effect of medication: a population-based study. JAMA Psychiatry. 2014;71(3):319-325. doi:10.1001/jamapsychiatry. 2013.4174

9. Banaschewski T, Johnson M, Lecendreux M, et al. Health-related quality of life and functional outcomes from a randomized-withdrawal study of long-term lisdexamfetamine dimesylate treatment in children and adolescents with attention-deficit/ hyperactivity disorder. CNS Drugs. 2014;28(12):1191-1203. doi:10. 1007/s40263-014-0193-z

10. Asherson P, Chen W, Craddock B, Taylor E. Adult attention-deficit hyperactivity disorder: recognition and treatment in general adult psychiatry. $B r \quad J$ Psychiatry. 2007;190(1):4-5. doi:10.1192/bjp. bp.106.026484

11. Coghill DR, Banaschewski T, Soutullo C, Cottingham MG, Zuddas A. Systematic review of quality of life and functional outcomes in randomized placebo-controlled studies of medications for attention-deficit/hyperactivity disorder. Eur Child Adoles Psy. 2017;26(11):1283-1307. doi:10.1007/s00787-017-0986-y

12. Quinn PD, Chang Z, Hur K, et al. ADHD medication and substance-related problems. Am J Psychiatry. 2017;174(9):877-885. doi:10.1176/appi.ajp.2017.16060686

13. Man KK, Coghill D, Chan EW, et al. Association of risk of suicide attempts with methylphenidate treatment. JAMA Psychiatry. 2017;74 (10):1048-1055. doi:10.1001/jamapsychiatry.2017.2183

14. Liang SHY, Yang YH, Kuo TY, et al. Suicide risk reduction in youths with attention-deficit/hyperactivity disorder prescribed methylphenidate: a Taiwan nationwide population-based cohort study. Res Dev Disabil. 2018;72:96-105. doi:10.1016/j.ridd.2017.10.023

15. Dalsgaard S, Hansen N, Mortensen PB, Damm D, Thomsen P. Reassessment of ADHD in a historical cohort of children treated with stimulants in the period 1969-1989. Eur Child Adoles Psy. 2001;10(4):230-239. doi:10.1007/s007870170012

16. Liao YT, Yang YH, Kuo TY, et al. Dosage of methylphenidate and traumatic brain injury in ADHD: a population-based study in Taiwan. Eur Child Adoles Psy. 2017:1-10. doi:10.1007/s00787-01 7-1042-7.

17. Chen VCH, Yang YH, Liao YT, et al. The association between methylphenidate treatment and the risk for fracture among young ADHD patients: a nationwide population-based study in Taiwan. PLoS One. 2017;12(3):e0173762. doi:10.1371/journal.pone.0173762

18. Rasmussen K, Palmstierna T, Levander S. Differences in psychiatric problems and criminality between individuals treated with central stimulants before and after adulthood. J Atten Disord. 2019;23 (2):173-180. doi:10.1177/1087054715571740

19. Cox NJ, Subbarao K. Influenza. Lancet. 1999;354(9186):1277. doi:10.1016/S0140-6736(99)01241-6
20. Neuzil KM, Wright PF, Mitchel EF, Griffin MR. The burden of influenza illness in children with asthma and other chronic medical conditions. J Pediatr. 2000;137(6):856-864. doi:10.1067/mpd.2000. 110445

21. Lafond KE, Nair H, Rasooly MH, et al. Global role and burden of influenza in pediatric respiratory hospitalizations, 1982-2012: a systematic analysis. PlosOne. 2016;13(3):e1001977. doi:10.1371/ journal.pmed.1001977

22. Warren-Gash C, Fragaszy E, Hayward AC. Hand hygiene to reduce community transmission of influenza and acute respiratory tract infection: a systematic review. Influenza Other Resp. 2013;7(5):7 38-749. doi:10.1111/irv.12015

23. Timpka T, Spreco A, Gursky E, et al. Intentions to perform non-pharmaceutical protective behaviors during influenza outbreaks in Sweden: a cross-sectional study following a mass vaccination campaign. PlosOne. 2014;9(3):e91060. doi:10.1371/journal.pone.009 1060

24. Tsai YY, Lee JJ, Hsieh WH. Determinants of the public intent to receive the seasonal influenza vaccine and protective behaviors: a population-based study in Taiwan. Vaccine. 2014;32(49):6667-6675. doi:10.1016/j.vaccine. 2014.08 .080

25. Shim E. Optimal strategies of social distancing and vaccination against seasonal influenza. Math Biosci Eng. 2013;10(5/6):16 15-1634. doi:10.3934/mbe.2013.10.1615

26. Schmitt J, Buske-Kirschbaum A, Roessner V. Is atopic disease a risk factor for attention-deficit/hyperactivity disorder? A systematic review. Allergy. 2010;65(12):1506-1524. doi:10.1111/j.1398-9995.20 10.02449.x

27. Chen $\mathrm{MH}, \mathrm{Su} \mathrm{TP}, \mathrm{Chen} \mathrm{YS}$, et al. Is atopy in early childhood a risk factor for ADHD and ASD? A longitudinal study. J Psychosom Res. 2014;77(4):316-321. doi:10.1016/j.jpsychores.2014.06.006

28. van der Schans J, Cicek R, de Vries TW, et al. Association of atopic diseases and attention-deficit/hyperactivity disorder: a systematic review and meta-analyses. Neurosci Biobehav Rev. 2017;74: 139-148. doi:10.1016/j.neubiorev.2017.01.011

29. Miyazaki C, Koyama M, Ota E, et al. Allergic diseases in children with attention deficit hyperactivity disorder: a systematic review and meta-analysis. BMC Psychiatry. 2017;17(1):120. doi:10.1186/s1288 8-017-1281-7

30. Kloepfer KM, Olenec JP, Lee WM, et al. Increased H1N1 infection rate in children with asthma. Am J Respir Crit Care Med. 2012;185 (12):1275-1279. doi:10.1542/peds.2013-2294CCC

31. Santillan Salas CF, Mehra S, Pardo Crespo MR, et al. Atopic conditions other than asthma and risk of the 2009 novel H1N1 infection in children: a case-control study. Allergy Asthma Proc. 2013;34 (5):459-466. doi:10.2500/aap.2013.34.3686

32. Lo PK Hand hygiene interventions and impacts on school children. HKU Theses Online (HKUTO); 2015.

33. Tzang RF, Chang YC, Hsu K. Factors influencing nail biting habit in children with ADHD. Int J Child Dev Mental Health. 2015;4 (1):7-16.

34. Ghanizadeh A. Association of nail biting and psychiatric disorders in children and their parents in a psychiatrically referred sample of children. Child Adol Psych Men. 2008;2(1):13. doi:10.1186/17532000-2-13

35. Lin LY, Warren-Gash C, Smeeth L, et al. Data resource profile: the National Health Insurance Research Database (NHIRD). Epidemiol Health. 2018;40:e2018062. doi:10.4178/epih.e2018062

36. American Psychiatry Association. Diagnostic and Statistical Manual of Mental Disorders: DSM-IV. Washington, DC: American Psychiatric Publishing; 1994.

37. American Psychiatry Association. Diagnostic and Statistical Manual of Mental Disorders: DSM-IV-TR ${ }^{\circledR}$. Washington, DC: American Psychiatric Publishing; 2000.

38. World Health Organization. International Statistical Classification of Diseases, Ninth Revision (ICD-9). Geneva; 1975. 
39. Lee M-J, Yang K-C, Shyu Y-C, et al. Attention-deficit hyperactivity disorder, its treatment with medication and the probability of developing a depressive disorder: a nationwide population-based study in Taiwan. J Affect Disord. 2016;189:110-117. doi:10.1016/j.jad.2015. 09.015

40. Mao L. Evaluating the combined effectiveness of influenza control strategies and human preventive behavior. PlosOne. 2011;6(10): e24706. doi:10.1371/journal.pone.0024706

41. Rewar S, Mirdha D, Rewar P. Treatment and prevention of pandemic H1N1 influenza. Ann Glob Health. 2015;81(5):645-653. doi:10.1016 j.aogh.2015.08.014

42. Oades RD, Dauvermann MR, Schimmelmann BG, Schwarz MJ, Myint AM. Attention-deficit hyperactivity disorder (ADHD) and glial integrity: S100B, cytokines and kynurenine metabolism-effects of medication. Behav Brain Funct. 2010;6:32. doi:10.1186/17449081-6-29

43. Dhabhar FS. Effects of stress on immune function: the good, the bad, and the beautiful. Immunol Res. 2014;58(2-3):193-210. doi:10.1007/ s12026-014-8517-0

44. Vitlic A, Lord JM, Phillips AC. Stress, ageing and their influence on functional, cellular and molecular aspects of the immune system. Age. 2014;36(3):9631. doi:10.1007/s11357-014-9631-6

45. Bigler MB, Egli SB, Hysek CM, et al. Stress-induced in vivo recruitment of human cytotoxic natural killer cells favors subsets with distinct receptor profiles and associates with increased epinephrine levels. PloSOne. 2015;10(12):e0145635. doi:10.1007/s11357-014 9631-6

46. Kreijtz JH, Fouchier RA, Rimmelzwaan GF. Immune responses to influenza virus infection. Virus Res. 2011;162(1-2):19-30. doi:10.10 16/j.virusres.2011.09.022
47. Rowe RK, Gill MA. Asthma: the interplay between viral infections and allergic diseases. Immunol Allergy Clin North Am. 2015;35 (1):115-127. doi:10.1016/j.iac.2014.09.012

48. Glanville N, Peel TJ, Schröder A, et al. Tbet deficiency causes T helper cell dependent airways eosinophilia and mucus hypersecretion in response to rhinovirus infection. PLoS Pathog. 2016;12(9): e1005913. doi:10.1371/journal.ppat.1005913

49. Lynch JP, Werder RB, Simpson J, et al. Aeroallergen-induced IL-33 predisposes to respiratory virus-induced asthma by dampening antiviral immunity. J Allergy Clin Immunol. 2016;138(5):1326-1337. doi:10.1016/j.jaci.2016.02.039

50. Centers for Disease Control, R.O.C (Taiwan). CDC Annual Report 2012; 2012. Available from: https:/www.cdc.gov.tw/english/infec tionreportinfo.aspx?treeid $=3847719104$ be 0678 \&nowtreeid= 73ce24f5d5c1fda4\&tid=B0B038F9F893517E. Accessed April 30, 2020.

51. Meyer D, Shearer MP, Chih YC, Hsu YC, Lin YC, Nuzzo J. Taiwan's annual seasonal influenza mass vaccination program-lessons for pandemic planning. Am J Public Health. 2018;108(S3):S188-S193. doi:10.2105/AJPH.2018.304527

52. Gau S, Chen SJ, Chou WJ, et al. National survey of adherence, efficacy, and side effects of methylphenidate in children with attention-deficit/hyperactivity disorder in Taiwan. J Clin Psychiatry. 2008;69(1):131-140. doi:10.4088/JCP.v69n0118

53. Moraes JC, Ribeiro MCSA. Social inequalities and vaccination coverage: utilization of household surveys. Rev Bras Epidemiol. 2008;11:113-124. doi:10.1590/S1415-790X2008000500011

54. Barbieri CL, Couto MT. Decision-making on childhood vaccination by highly educated parents. Rev Saude Publ. 2015;49:18. doi:10. 1590/S0034-8910.2015049005149
Neuropsychiatric Disease and Treatment

\section{Publish your work in this journal}

Neuropsychiatric Disease and Treatment is an international, peerreviewed journal of clinical therapeutics and pharmacology focusing on concise rapid reporting of clinical or pre-clinical studies on a range of neuropsychiatric and neurological disorders. This journal is indexed on PubMed Central, the 'PsycINFO' database and CAS, and is the official journal of The International Neuropsychiatric Association (INA). The manuscript management system is completely online and includes a very quick and fair peer-review system, which is all easy to use. Visit http://www.dovepress.com/testimonials.php to read real quotes from published authors. 